\title{
BMJ Open Exploring the role of social capital, self- efficacy and social contagion in shaping lifestyle and mental health among students representing the future healthcare workforce in Palestine: social cohort study protocol
}

\author{
Ranin A M Darkhawaja (10 ,1,2 Marek Kwiatkowski, ${ }^{1,2}$ Thomas Vermes, ${ }^{1,2}$ \\ Hala Allabadi, ${ }^{3}$ Sonja Merten, ${ }^{1,2}$ Abdulsalam Alkaiyat, ${ }^{3}$ Nicole Probst-Hensch ${ }^{1,2}$
}

To cite: Darkhawaja RAM Kwiatkowski M, Vermes T, et al. Exploring the role of social capital, self-efficacy and social contagion in shaping lifestyle and mental health among students representing the future healthcare workforce in Palestine: social cohort study protocol. BMJ Open 2022;12:e049033. doi:10.1136/ bmjopen-2021-049033

- Prepublication history for this paper is available online. To view these files, please visit the journal online (http://dx.doi. org/10.1136/bmjopen-2021049033).

Received 17 January 2021 Accepted 28 0ctober 2021

Check for updates

(c) Author(s) (or their employer(s)) 2022. Re-use permitted under CC BY-NC. No commercial re-use. See rights and permissions. Published by BMJ.

For numbered affiliations see end of article.

Correspondence to Dr Nicole Probst-Hensch; nicole.probst@swisstph.ch

\section{ABSTRACT}

Introduction Non-communicable diseases (NCDs) and depression form an unhealthy mix. The project focuses on potentially effective psychosocial factors shaping healthrelated habits and mental health. The study is conducted among health domain students. Understanding what shapes their health will determine their quality of care. The study is implemented at An-Najah National University in Palestine. This zone of continuous conflict psychological stress is high and mental health problems are stigmatised. Methods and analysis Students who are enrolled in second and third year will be invited to fill in a baseline and two follow-up online questionnaires. The questionnaires will assess: health behaviours and outcomes (health-related habits, obesity and mental health), main predictors (social capital, social network, self-efficacy), confounders (general and sociodemographic characteristics) and effect modifiers (sense of coherence (SOC) and family SOC). Friendships within participating students will be identified by allowing students to name their friends from a pull-down menu of all students. Descriptive statistics and scores will describe participant's characteristics. The relationship between health behaviour, outcomes and main predictors will be examined by regression and structural equation models. Clustering of health behaviours and outcomes will be assessed by permutation tests. Their spread within the network of friends will be investigated by longitudinal generalised estimating equations.

Discussion The study will identify the prevalence of NCDrelated health habits and mental health aspects in the future healthcare workforce in Palestine. It will be the first study to address the role of psychosocial factors for the targeted students. It has the potential to identify targets for promoting physical and mental health among these future professionals.

Ethics and dissemination Ethical approval was obtained from Ethikkommission Nordwest- und Zentralschweiz (EKNZ) in Switzerland and the Institutional Review Board Committee (IRBC) in Palestine. Participation in the study is voluntary and requires informed consent. The data
Strengths and limitations of this study

- As one of the rare studies to longitudinally address the role of psychosocial factors including social contagion in shaping the lifestyle and mental health among network ties of students, it may identify novel cost-effective preventive targets.

- By focusing on health domain students in a geographical area of long-term conflict that generally promotes unhealthy habits and aggravates mental ill-health, the study offers novel insight on how to promote prevention knowledge, acceptance and skills of the future healthcare workforce.

- The analysis of the longitudinal directional network ties and attributes of the study participants uncover and quantify the suspected social contagion of health-relatedbehaviours and mental health.

- The sample will be taken only from An-Najah National University. The results of the study will, therefore, not be representative of all health domain students in Palestine.

- The study will be based on self-report tools and restricted to friendship nominations among the student network. This can lead to misclassification of characteristics, in particular, those associated with some stigma including mental health symptoms.

management methodology ensures the confidentiality of the data. The outcomes of the study will be published as scientific papers. In addition, it will be presented in stakeholder conferences and to students at An-Najah National University.

\section{INTRODUCTION}

\section{Background}

Non-communicable diseases (NCDs) are major contributors to morbidity and mortality worldwide. ${ }^{1}$ Globally, NCDs accounted for more than $70 \%$ of all deaths in $2017 .^{2}$ In 2019 , 
NCDs were also the leading causes of death in Palestine. Cardiovascular diseases followed by cancer accounted for $29.9 \%$ and $15.5 \%$ of total deaths, respectively. ${ }^{3}$ Research studies reported a high prevalence of important NCD risk factors such as tobacco smoking, ${ }^{4}$ waterpipe smoking, ${ }^{5}$ alcohol drinking, use of illicit drugs, ${ }^{6}$ low adherence to healthy nutrition ${ }^{48}$ and low physical activity (PA) ${ }^{79}$ among Palestinian youths. Because NCDs evolve over the course of life and because lifestyle tracks from young to old age, young Palestinians put themselves at high risk of developing NCDs later in life by adopting an unhealthy lifestyle at young age.

In Palestine, there is a high prevalence of mental health problems. Poor mental health is a major driver of disability. ${ }^{10}$ Despite this, there is still a gap in the recognition, diagnosis and treatment of mental disorders in Palestine. ${ }^{11}$ Unaddressed mental care needs are an important barrier to the successful control of NCDs in low and middle-income countries. ${ }^{12}$ The relationship between the most common NCDs (cardiovascular diseases and diabetes) and depression is thought to be bidirectional. ${ }^{13}$ Alarmingly, high rates of severe depression and anxiety were reported among Palestinian cardiac patients. ${ }^{14}$

Based on Antonovsky's salutogenenesis theory, the current study focuses on the role of individuals' psychosocial factors in shaping their lifestyle and mental health. According to the theory, at any point in time, an individual has a position on the generalised resistance resources-resistance deficits (GRRs-RDs) continuum. ${ }^{15}$ Accessibility of resistance resources along the continuum allows the individual to experience 'consistency' in life, to have 'load balance' between resources and demands, and to 'participate in decision-making'. In turn, these experiences allow the individual to develop a sense of coherence (SOC) ${ }^{15-17}$ SOC development contributes to the individual's mental and physical health through its ability to effectively mobilise resources for overcoming stressors and challenges and ensuring positive health and health habits. ${ }^{1516} 18$ Social capital (SC) is hypothesised to be one of the GRRs through which SOC can act as a mediator between SC and health outcomes. ${ }^{19}$ There is evidence for the positive association of network capital $^{20-23}$ and cognitive $\mathrm{SC}^{20-22 ~ 24-27}$ with both, physical and mental health. In addition, there are areas of convergence between self-efficacy (SE) and SOC. SE is characterised by being consistent, by contributing to shaping outcome expectancies and behaviour and by leading to overload and underload balance. Thus, it appears to be one of the GRRs, which contributes to the enhancement of SOC. ${ }^{1728}$ There is evidence for an association between SE and health-related behaviours. ${ }^{17}{ }^{28}$ Also, SE is positively associated with perceived good health, well-being and quality of life, ${ }^{17}$ but inversely with depression and anxiety. ${ }^{29}$

This study is based on the assumption that SC and SE contribute to the development of SOC, which in turn influences individuals' health-related behaviours and mental health. See figure 1 for reference.

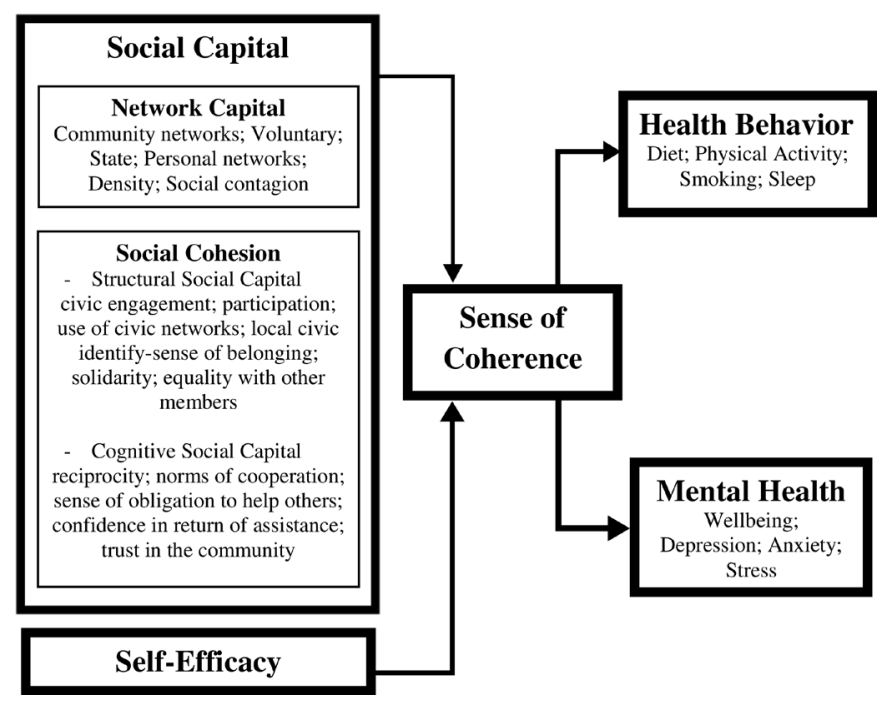

Figure 1 Conceptual framework of the study based on the Antonovsky's theory of salutogenesis.

Furthermore, the social contagion theory suggests that behaviours can spread from one person to another within social networks through induction. ${ }^{30}{ }^{31}$ This is evidenced by the clustering of smoking and obesity within social networks. ${ }^{31}{ }^{32}$ There is also evidence for the contagion of mental health through the spread of happiness ${ }^{33}$ and depression ${ }^{34}$ within social networks.

Psychosocial factors and social contagion may be important and cost-effective targets for more efficient NCD prevention and mental health promotion in Palestine. The continuous conflict in this region contributes to poor health habits and to mental problems, but the later are not properly addressed due to cultural stigmatisation. Healthcare professionals own health, mental health, habits and perceptions play an important role in how they provide care, including preventive and mental health counselling. ${ }^{35}{ }^{36}$ It is, therefore, important to study health habits and mental health as well as their psychosocial contributors in health domain students. They are the future healthcare workforce, but they are still at an age where habits and perceptions are more easily modified. ${ }^{19}$

\section{Aim}

The aim of the study is to explore the role of SC, SE, SOC and social contagion in shaping lifestyle and mental health among health domain students, representing the future healthcare workforce in Palestine.

\section{Specific objectives}

The study will be conducted among students enrolled in the faculty of medicine and health sciences of An-Najah National University to:

1. Assess the frequency, distribution and clustering of self-perceived health, health-related behaviours, obesity and mental health among students.

2. Assess the distribution of SC, SE and SOC among students. 
3. Estimate the association between SC, SE and SOC with the longitudinal course of self-perceived health, health-related behaviours, obesity and mental health among students.

4. Uncover and quantify the suspected social contagion of health-related behaviours, obesity, and mental health among students.

5. Identify modifying and mediating factors in the association of SC and SE with the level and longitudinal course of self-perceived health, health-related behaviours, obesity and mental health among students.

\section{METHODS AND ANALYSIS \\ Study population \\ Inclusion criteria}

The study will invite all second and third-year students enrolled in the optometry (104 students), medical imaging (110), nursing (480), pharmacy (304), medicine (331), physiotherapy (122), biomedical sciences I (739), medical laboratory (345), midwifery (29), doctor of pharmacy (122) and audiology and speech therapy (157) programmes of the faculty of medicine and health sciences of An-Najah National University, Nablus, Palestine. First-year students will be excluded from the study because friendship ties among university students are more likely to start evolving during the first academic year of enrolment and develop by the second academic year of enrolment. Also, first-year students are less likely to continue studying. Senior students (fourth, fifth and sixth-year students) are excluded because the minimum number of years required to complete programmes in the faculty of medicine and health sciences is 4 years. Furthermore, inclusion of senior students harbours the risk of high attrition of the participants due to graduation from the university. The data collection will continue over the course of 1 year

\section{Recruitment}

A promotion video will be shared with the targeted students to introduce the study and encourage them to take part in the survey. Students will obtain personal invitations for study participation by email. The email will include a personalised link to access online study information, e-consent form and questionnaires. Short Message Service (SMS) messages and emails will be sent to the students as reminders to complete the survey. After the end of the study, an event will be organised with the aim of increasing the students' understanding and awareness on aspects related to their future professional career. The study's result will form the basis for input into this event.

\section{Study design}

The study is a digital prospective cohort; it is based on online baseline survey and two follow-up surveys (first follow-up 6 months after baseline and second follow-up 12 months after baseline) surveys. The study will be conducted between 2021 and 2022.

\section{Data collection and management}

Data will be collected with a culturally sensitive online questionnaire. Questionnaires will be developed in English and will be translated to Arabic and backtranslated to English. The questionnaires will be piloted among a subset of first-year students enrolled at the faculty of medicine and health sciences of An-Najah National University, Nablus, Palestine.

Data recording will be performed in the data collection tool REDCap, a secure web application for building and managing online surveys and databases. The questionnaire will be administered three times. For each of the baseline and follow-up surveys, students will be given 30 days to complete the survey, starting from the day they receive the invitation.

An-Najah National University will provide Swiss Tropical and Public Health Institute (Swiss TPH) with emails and names of the eligible students. An open personidentifying ID (OID) will be assigned to each student. First, a personalised link (OID as identifier for the link) will be mailed to students. Both, student's email and the student portal 'Zajel' will be used for this mailing. The link will allow the students to access and fill in the participant information sheet, the contact information sheet and the e-consent. Second, after the students provide their e-consent and confirm their personal information, they will receive an email with a personalised link for accessing online questionnaires.

The completed online research questionnaire (research data) will be stored in REDCap. To assure a high quality of the data, the records of the survey in REDCap will be checked daily by the responsible researcher. Students will be immediately reconnected to complete missing data. At the end of data collection, the research data will be pseudonymised with each student being assigned a research ID Pseudonymisation ID (PID). The students' personally identifying information (first name, father name, grandfather name and last name, date of birth, gender, student domain, year of the study, student ID and student email) will be stored in a separate file in REDCap, linked to OID. In addition, a link file between OID and PID will be stored separately as a file outside REDCap. The files with person-identifying data and the link file will only be accessible to the principal investigator of the study (NP-H) and the chief security officer of Swiss TPH. The social network of a student (friends and colleagues) will be identified in the research database as friends' and colleagues' PIDs. After completion of the study and by 31 December 2023, any information that would identify the participant (first name, surnames, date of birth, gender, student domain, year of the study, student ID and student email) will be deleted from REDCap. The link file between OID and PID will be deleted in order to fully anonymise the research data. The anonymised research data of the study along with the e-consent forms will be stored on servers of Swiss TPH in Switzerland. 


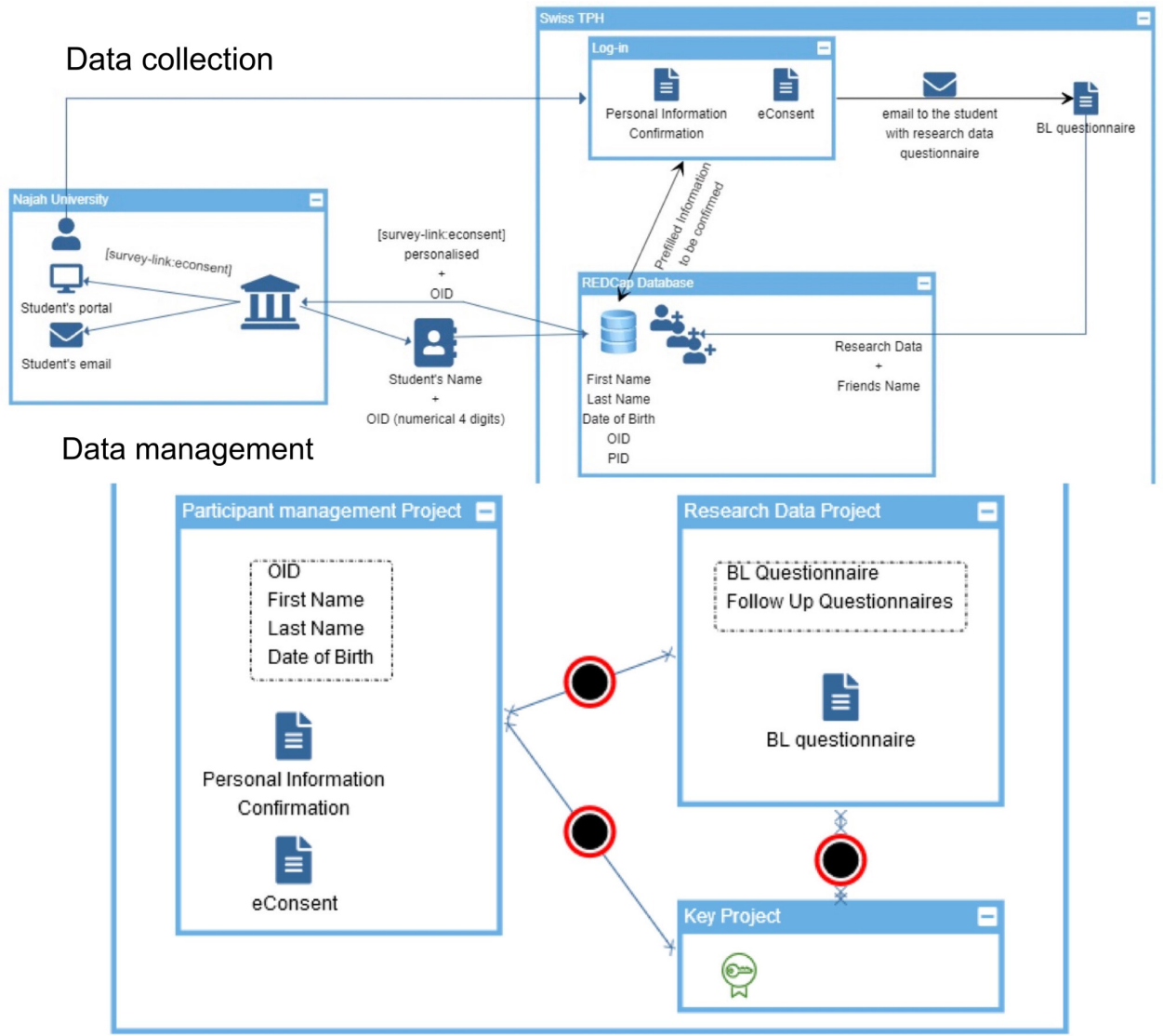

Figure 2 Data collection and management procedure. BL, baseline; OID, open person-identifying ID; PID, Pseudonymisation ID.

The procedure for administrating the first and second follow-up surveys will be the same as administrating the baseline survey.

Students and their personal identifying information will only be retained in the participant management file if they provided consent for study information. Only students who completed the previous survey will be targeted for the next survey. See figure 2 for reference.

\section{Measurements}

Primary endpoint

Health-related habits

Diet

1. Food intake: the intake frequency of 14 items will be measured; fruits, vegetables, dried fruits, poultry, meat, fish $\mathcal{E}$ seafood, instant noodles, soft drinks, sweetened juice, cake $\mathcal{E}$ sweets $\mathcal{E}$ chocolate $\mathcal{E}$ biscuits, chips $\mathcal{E} \odot$ salty snacks, fast food, caffeinated beverages and energy drinks. This will ensure the assessment of students' dietary intake according to the WHO dietary guidelines recommendations for the Eastern Mediterranean region. ${ }^{37}$

2. Drinking water: the quantity and the type of drinking water will be assessed.

The food intake will be categorised into healthy and unhealthy dietary intake based on the WHO dietary guidelines recommendations for the Eastern Mediterranean region. ${ }^{37}$ One point will be given if the food frequency intake is in line with the WHO dietary guidelines recommendations for the Eastern Mediterranean region. See table 1 for more details.

A score of 6 or more total points will be considered as 'healthy dietary intake', a score below 6 points as 'unhealthy dietary intake'.

3. Weight loss: participants will be asked if they have tried to lose weight in the past. The relation of perception of weight loss with participant's own body mass index (BMI) on the one hand, and with attempts to lose weight in the participant's student network will be investigated.

\section{PA and sedentary behavior}

PA in the past 7 days will be assessed with the short version of the international physical activity questionnaire (IPAQ), which will be included in the questionnaire. Participants' PA will be estimated based on the frequency 'days per week' and duration 'hours/min spent" spent in three activity domains: walking, moderate PA and vigorous PA. ${ }^{38}$ Sedentary activity of the participants will be assessed separately for weekend days and weekdays. Scores will be calculated for each PA intensity level and converted into metabolic equivalent (MET) according to the IPAQ scoring protocol. Minutes per week will be converted to MET using the conversion factor 3.3 for walking, 4.0 for moderate PA and 8 for vigorous PA. The total score of PA will be categorised into low PA, moderate PA and high PA. ${ }^{38}$ 
Table 1 WHO dietary guidelines recommendations for Eastern Mediterranean region

\begin{tabular}{ll}
\hline Item & $\begin{array}{l}\text { Recommended food } \\
\text { frequency intake }\end{array}$ \\
\hline Health items & $\begin{array}{l}\text { Several times per week, daily } \\
\text { or several times per day }\end{array}$ \\
\hline $\begin{array}{l}\text { Fruits } \\
\text { Vegetables }\end{array}$ & Several times per week \\
\hline $\begin{array}{l}\text { Dried fruits } \\
\text { Poultry }\end{array}$ \\
$\begin{array}{l}\text { Meat } \\
\text { Fish and seafood }\end{array}$ \\
$\begin{array}{l}\text { Water } \\
\text { Unhealthy items }\end{array}$ \\
$\begin{array}{l}\text { Instant noodles } \\
\text { Soft drinks }\end{array}$ \\
$\begin{array}{l}\text { Sweetened juice } \\
\text { Cake and sweets and chocolate } \\
\text { and biscuits } \\
\text { Chips and salty snacks } \\
\text { Fast food } \\
\text { Caffeinated beverages } \\
\text { Energy drinks }\end{array}$ \\
\hline
\end{tabular}

\section{Tobacco use}

The history of and the current tobacco use (cigarettes, hookah/shisha and electronic cigarettes) among participants will be assessed in addition to the participant's attempt to quit smoking.

\section{Sleep pattern}

It will be measured with the help of the Pittsburgh Sleep Quality Index (PSQI). ${ }^{39}$ Global PSQI score ranging from 0 to 20 will be calculated. A global score $>5=$ poor quality of sleep and $\leq 5$ =good quality of sleep. ${ }^{39}$

\section{Obesity}

The participants will be asked to measure or self-report their weight and height. According to the WHO classifications of BMI for adults, participants will be categorised as underweight $\left(\mathrm{BMI}<18.5 \mathrm{~kg} / \mathrm{m}^{2}\right)$, normal weight $\left(18.5-24.9 \mathrm{~kg} / \mathrm{m}^{2}\right)$, overweight (BMI $\left.25-29.9 \mathrm{~kg} / \mathrm{m}^{2}\right)$ and obese $\left(\mathrm{BMI} \geq 30 \mathrm{~kg} / \mathrm{m}^{2}\right) .{ }^{40}$ It will be recorded whether the reported height and weight were measured or not.

\section{Self-perceived health}

It will be evaluated by asking the participant about their experience of chronic health condition or disability.

\section{Mental health}

\section{Well-being}

Most of the existing research focuses on the negative aspects of mental health including depression. ${ }^{19}$ Thus, the assessment of well-being is important to explore the relationship between the psychosocial factors (SC, SE and SOC) and health-related habits with the positive aspect of mental health. Moreover, based on the salutogenesis theory, well-being is one component of health, which is shaped by psychosocial factors. ${ }^{15}$ Well-being will be rated by using the WHO five well-being index. ${ }^{41}$ The total score will range from 0 to $100(\geq 50=$ moderate to high level of well-being; $<50=$ ill-being).

\section{Depression, anxiety and stress}

These are negative aspects of mental health. They will be measured by applying the depression, anxiety and stress scale (DASS 21). ${ }^{42}$ The DASS-21 scale assesses each of DASS based on seven items. Each item is scored on a 0-4 scale: ' never $=0$, sometimes $=1$, often $=2$, almost $=3$ and always $=4$ '. The score for each domain will be calculated by summing up the scores for the corresponding seven items and multiplying the total by 2 . Then, the score will be divided into the following categories (normal=0-9, mild $=10-$ 13, moderate $=14-20$, severe $=21-27$ and extremely severe $=28+$ ) for each domain.

\section{Main predictors \\ Social capital}

SC will be measured using the SC questionnaire developed by Yari et $a l^{43}$

\section{Social network}

The social contagion of health-related behaviours, obesity and mental health will be assessed by collecting information on the close friends/colleagues of each student. An-Najah National University will provide a list of the name of all students enrolled at the faculty of medicine and health sciences. Additionally, the list will include the main sociodemographic characteristics of the students (gender, date of birth, programme name and academic year). The survey will include a drop down menu of the student's list obtained from the university. The participants can mark their close friend in this list. Because the list includes all potential study participants, it allows for the assessment of social contagion.

\section{Self-efficacy}

Both general SE and social SE among the students will be measured based on Sherer eta al's scale. ${ }^{44}$

\section{Confounders, effect modifiers}

\section{General and sociodemographic characteristics}

This section inquires about gender, age, marital status, religion, place of residence, housing facility, refugee status, household monthly income, size of household, employment status, parent's occupations, care provision for others, affiliated faculty, current academic year.

\section{Mediators}

Sense of coherence

It will be assessed based on Antonovsky's SOC scale. ${ }^{45}$ The scale consists of 13 items. Each item is scored from 1 to

7. Thus, the scale will have a maximum score of 91 and a minimum score of 13 , with high scores indicating high SOC. 
Family SOC

It will be assessed based on Shifra Sagy's scale. ${ }^{46}$ The scale consists of 12 items. Each item is scored from 1 to 7. Thus, the scale will have a maximum score of 84 and a minimum score of 12 . High scores indicate perception of better family life coherence.

\section{Statistical analysis}

\section{Sample size and characteristics}

Assuming a 50\% participation rate, we aim to achieve a sample size of $\mathrm{n}=1000$, which is sufficient to estimate prevalence of any binary trait with a margin of error of at most 3.1 percentage points. We expect that $50 \%$ of the students do not exhibit healthy habits and $20 \%$ have at least moderate depression symptoms. If we dichotomised a predictor such as SC along the median, this sample would confer $85 \%$ power to detect at the $5 \%$ significance level risk differences of 8 percentage points for depression and 10 percentage points for unhealthy lifestyle between the low and high SC groups. We anticipate having similar power to detect considerably smaller effects when analysing continuous outcomes and pooling data across survey waves. We have forgone formal power calculations for the clustering and contagion objectives because they depend critically on network topology, for which we have no prior information.

Descriptive statistics (n, \%, mean) will describe participant's general and sociodemographic characteristics as well as tobacco use, obesity and self-perceived health. Scores will describe diet, PA, sleep pattern, happiness, loneliness, well-being, depression, anxiety, stress, SC, social network, SE and SOC. The longitudinal associations between primary endpoints and main predictors will be studied using mixed linear and logistic regression models. Interaction terms will be included in the models to capture effect modification. Mediation will be assessed by structural equation modelling.

\section{Non-response and attrition}

We acknowledge the potential of non-respondent and attrition given the longitudinal design of the study. This can affect the generalisability of the outcomes due to selection bias and decrease the statistical power of the study. ${ }^{47-49}$ Several mitigation strategies will be applied to account for potential non-respondent and attrition among participants. First, at the recruitment level, the targeted study population was selected to maximise both participation and retention rates. The study population are second and third-year students of the faculty of medicine and health sciences of An-Najah National University, Palestine. The minimum number of years to complete any programme in the faculty of medicine and health sciences is 4 years and the tenure of data collection will be 1 year. This will avoid the possibility of loss to follow-up due to graduation and absence of any connection to the university. Second, incentives will be provided for participants with the condition of completing the baseline and two follow-up surveys. Additionally, reminders will be sent to the students through emails and text messages to increase participation rate and retention of the participants. Furthermore, a promotion video will be shared with the targeted students to introduce the study and encourage them to take part in the survey. These strategies proved to increase participation rate and maximise retention rate in cohort studies. ${ }^{48}$ At the statistical analysis level, we have access to basic demographic characteristics of all the study population. This will allow us to make comparisons between participants and non-participants at baseline and to assess if there are significant differences between them. Analysis of non-respondents will help establish who will be at high risk of attrition in the future wave. ${ }^{48}$ Subsequently, strategies to increase the retention rate of such participants will be implemented. ${ }^{40}{ }^{50}$ Inverse probability weighting will be applied to ensure that the analyses are unbiased in spite of attrition. ${ }^{485152}$

\section{Clustering of health behaviours and outcomes in social networks}

The relationships between the study participants will be captured in a network: each participant will be represented as a node that is linked to other nodes according to the friendship information provided in the surveys. We will distinguish between directed links, when one participant nominates another as a friend but not vice versa, and undirected links, when nomination was mutual. Thus, three types of relationships are possible between any pair of nodes ('ego' and 'alter'): ego-perceived friendship, alter-perceived friendship and mutual friendship. We will perform our analyses separately for each type. We will also be able to follow the evolution of this network across time due to the follow-up surveys.

Our statistical approach is based on that of Christakis and Fowler. ${ }^{31}$ We will use permutation tests to assess clustering of the primary outcomes in the network. Every outcome will be dichotomised. The degree of clustering will be characterised by the relative increase in the probability (ie, risk ratio) that an ego has the dichotomous trait of interest given that an alter has it, compared with the probability that an ego has the trait given that an alter does not have it. This value computed for the observed network will be compared with that of computergenerated networks of the same topology. In the later, the trait is distributed uniformly randomly across the nodes while keeping the overall observed prevalence. If the risk ratio in the observed network is greater than in $95 \%$ or more of the synthetic networks, then the hypothesis that the observed clustering is entirely due to chance will be rejected at the $5 \%$ significance level.

\section{Social contagion analysis}

Longitudinal regression models will be used to assess the spread of health outcomes and health-related behaviours across network ties. The status of ego at first follow-up (respectively, second follow-up) will be expressed as a function of the ego status, alter status and covariates at baseline (respectively, first follow-up). Thus, each node in the network (ego) will contribute as many observations as 
there are nodes (alters) that it is connected to. The models will be estimated using generalised estimating equations with clustering on ego to take into account multiple observations of the same participant across surveys and alters. The fitted coefficient of the alter's status at baseline will be interpreted as an estimate of the intensity of spread. In particular, we will consider an outcome or a behaviour to have spread through the network if this coefficient is significantly different from 0 .

\section{Patient and public involvement}

It was not appropriate or possible to involve patients or the public in the design, or conduct, or reporting, or dissemination plans of our research.

\section{DISCUSSION}

In 2015, the UN Member States have adopted the target to reduce the premature mortality from NCDs by onethird by 2030 to achieve the third sustainable development goal (SDGs). ${ }^{53}$ Prior to this, in 2013, the World Health Assembly adopted the WHO Global Action Plan for the Prevention and Control of NCDs 2013-2020. It aims to accomplish 25\% relative reduction in overall mortality caused by major NCDs. ${ }^{1}$ Along with that WHO has recognised mental disorders as one of the major NCDs. ${ }^{54}$ Depression is expected to become the main cause of disability globally. ${ }^{9}$ In the Global South, resource allocation for mental disorders is sparse, partly reflecting their stigmatisation. The Lancet Commission on Global Mental Health and SDGs is pointing to the importance of integrating mental health services with care for NCDs. ${ }^{55}$

In Palestine, on few and insufficient reports describe the size of the NCD problem. Complete national data on the burden of NCDs do not exist. ${ }^{56}$ There is still gap in the recognition, diagnosis and treatment of mental disorders in Palestine despite their high prevalence. ${ }^{10}{ }^{11}$ For example, despite the well-known bidirectional association between depression and cardiovascular disease, none of the cardiac patients treated in the main Nablus hospitals was ever assessed for depression by their treating cardiologists. ${ }^{14}$ The youth sector in Palestine is important as it accounts for a little less than quarter of the total population. ${ }^{57}$ The health needs of young Palestinians should be addressed as a matter of urgency.

To the best of our knowledge, no study has addressed the role of SC, SE SOC and social contagion in the health and well-being of the youth in Palestine. This study will target medical and other health domain students. They are also at risk of adopting risky health and lifestyle habits, particularly in the light of the challenges they face during their tenure of study. ${ }^{58}$

The assessment of self-perceived health, health-related behaviours, obesity and mental health is important as a way to identify health problems and modifiable risk factors (dietary risks, use of tobacco and low PA) among the students. The participating students are the future health professionals. Their health behaviours and mental health will shape the healthcare they will provide and their impact as role models in the society. ${ }^{59}$ There is evidence for the positive association between poor well-being ${ }^{6061}$ and sleep-related impairment among physicians ${ }^{62}$ and the increase in medical errors. ${ }^{60-62}$ Also, stress among healthcare provider is associated with an increase in the odds of medical errors. ${ }^{63}$ Physicians' positive well-being is associated with patient satisfaction and quality of patient care. ${ }^{64}$ Furthermore, this study can increase their awareness of healthy habits and healthy minds. This can contribute to their perception of primary prevention as a cost-effective approach to NCDs and it can promote the destigmatisation of mental health problems among these future healthcare professionals. ${ }^{65}$ Healthcare providers are not only the most credible source of prevention and health promotion counselling but also they have ethical obligations to provide counselling to their patients. ${ }^{35}$ There is evidence for the positive association between the healthcare provider's personal habits and their practice of counselling to patients. ${ }^{35}$ This positive association has been found in relation to a wide range of health-related and prevention practices, including PA, smoking, nutrition, vaccination and screening. ${ }^{36}$ This is confined not only to practicing physicians but also to medical students ${ }^{3566}$ in both developed and developing countries. ${ }^{67}$ Patient's adherence to preventive and health promotion counselling is also enhanced if it is rooted in the health provider's personal experience. ${ }^{36}$

Our study will estimate the association between SC, SE and SOC with the longitudinal course of self-perceived health, health-related behaviours, obesity and mental health among students. This will be based on the Antonovsky's Salutogenic Model of Health (SMH) theory. The theory resonates with the health promotion framework, which focuses on the individual's ability to control different determinants of health through their own resources, processes and outcomes in order to attain health regardless of what is offered by the healthcare system. ${ }^{16}$ Salutogenesis does not necessarily eradicate diseases such as NCDs; instead, it enhances the individual's ability to manage them well. In turn, it enhances well-being. ${ }^{68}$ Thus, the study will identify another potential target for future health intervention strategies. SE and SOC proved to be effective in the implementation of health promotion strategies because of their ability to detect, predict and change health status. ${ }^{17} \mathrm{SE}$ is not confined to the ability to change behaviour. It extends to the belief in the ability to accomplish social changes and to overcome the structural determinants of health. ${ }^{69}$ In addition, SE contributes to the increase in the individual's resilience against dangerous and stressful situations and protects against trauma. ${ }^{70}$ This is important for Palestine, as the psychosocial determinants of NCDs are influenced by the presence of the Israeli occupation that has been contributing to the political instability, movement restriction and violence. ${ }^{71}$ The daily stresses due to economic hardship, unemployment, family conflict, lack of public spaces for recreation and leisure and lack of 
sustainable facilities for physical activities, all increase the stress and ultimately their impact on NCD risk. ${ }^{71}$ Interventions to promote healthy behaviours among students were positively associated with their prevention-related attitudes and counselling practices. ${ }^{35} 667273$ Thus, appropriate interventions can be implemented to improve the health and well-being of the students.

The proposed study has several strengths. First, by focusing on Palestine where poor health habits and mental stressors are highly prevalent due to the ongoing conflict, cost-effective interventions to strengthen primary prevention and mental health promotion are much needed. From the study on the spread of health habits and mental health symptoms in the social network of students novel interventions can be derived.

The limitations of the study include, first, bias due to non-participation, which is of particular concern if students with few friends, poor health habits or poor mental health are not participating. This would lead to a lower appreciation of the role of psychosocial factors as determinants of healthy habits. Second, as any longitudinal study, loss to follow-up can bias the outcomes, particularly, if it is differential. Third, the prevalence of factors considered less healthy/unhealthy may be underestimated if students tend to underreport them for reasons of stigma. Fourth, despite the longitudinal nature of the study, three measurement time points may not be sufficient to properly characterise the short-term fluctuation of health habits and mental health symptoms. If this fluctuation is high and not captured, it potentially inhibits the ability of the study to identify associations if present.

\section{Ethics and dissemination}

The research protocol was approved by the Ethikkommission Nordwest- und Zentralschweiz (EKNZ) (AO_202000047) in Switzerland and the Institutional Review Board Committee at An-Najah National University (Ref: Med Nov. 2020/32).

Participation in the study is voluntary and participants will provide written informed e-consent. The students have the right to withdraw from the study at any time.

It is important to maintain the privacy of students and the information provided due to the high sensitivity. The proposed methodology ensures the confidentiality of the data as the study will be conducted in compliance with the current version of the Declaration of Helsinki as well as all national legal and regulatory requirements. During the active phase of data collection, participants will be identified by an open ID, which links to their person-identifying data and serves as personalised link to access study information and e-consent. Research data derived from each study follow-up will be stored with a separate research ID and will not contain person-identifying information. The link file between the person-identifying information and the research ID will only be accessible to a small and well-defined group of persons. After finalisation of the study following the second follow-up and by 31 December
2023, any information that would identify the participant (names, birth date, student ID) will be deleted for a fully anonymised research database.

The students who complete the survey will be rewarded with scoring points related to the student unions and non-governmental organisations. These scoring points give the students the opportunity to participate in events and projects organised by the university such as 'receiving scholarship to conduct scientific research'. In addition, the names of students who complete the survey will be entered into a drawing for a chance to win a coupon. Four names will be selected.

The outcomes of the study will be published as scientific papers. In addition, it will be presented in stakeholder conferences and to students at An-Najah National University.

\section{Author affiliations}

${ }^{1}$ Department of Epidemiology and Public Health, Swiss Tropical and Public Health Institute, Basel, Switzerland

${ }^{2}$ Department of Public Health, University of Basel, Basel, Switzerland

${ }^{3}$ Faculty of Medicine and Health Sciences, Al-Najah National University, Nablus, State of Palestine

Contributors NP-H developed the idea for the study. RAMD, MK, SM and NP-H developed the study concept, the study protocol and the study objectives. RAMD, AA, HA and NP-H developed the study instruments. RAMD and MK developed the statistical analysis approach. RAMD, TV, AA and NP-H developed the data management approach and the RedCap database. RAMD, AA and NP-H wrote the ethics protocol. RAMD, TV, MK and NP-H drafted the manuscript. All authors read, commented and approved the manuscript.

Funding This project has received funding from the European Union's Horizon 2020 research and innovation programme under the Marie Skłodowska-Curie grant agreement number 801076, through the SSPH+Global PhD Fellowship Programme in Public Health Sciences (GlobalP3HS) of the Swiss School of Public Health. In addition to receiving funding from the Swiss Government Excellence Scholarship, GlobalP3HS Programme and the Swiss Government Excellence Scholarship (Grant/ Award Number: N/A) are funding the salary of the PhD student. The GlobalP3HS Programme is also providing operational funds. Swiss TPH is providing funds for administrative and statistical support and for hosting the database-REDCap. An-Najah National University is covering the costs for contacting students through emails and SMS messages in order to recruit the students to participate in the study and send reminders to complete the surveys.

\section{Competing interests None declared.}

Patient and public involvement Patients and/or the public were not involved in the design, or conduct, or reporting, or dissemination plans of this research.

\section{Patient consent for publication Not applicable.}

Provenance and peer review Not commissioned; externally peer reviewed.

Open access This is an open access article distributed in accordance with the Creative Commons Attribution Non Commercial (CC BY-NC 4.0) license, which permits others to distribute, remix, adapt, build upon this work non-commercially, and license their derivative works on different terms, provided the original work is properly cited, appropriate credit is given, any changes made indicated, and the use is non-commercial. See: http://creativecommons.org/licenses/by-nc/4.0/.

\section{ORCID iD}

Ranin A M Darkhawaja http://orcid.org/0000-0002-2785-7237

\section{REFERENCES}

1 World Health Organization. Noncommunicable diseases country profiles 20182018

2 Institute for Health Metrics and Evaluation (IHME). GBD Seattle, WA: University of Washintgon, 2016. Available: https://vizhub.healthdata. org/gbd-compare/ 
3 Palestinian Ministry of Health, Center PHI. Health annual report, Palestine 20182019

4 Al-Sabbah $\mathrm{H}$, ed. Overweight and obesity among university students in Palestine. International Conference and Exhibition on Nutritional Science and Therapy, 2012.

5 Tucktuck M, Ghandour R, Abu-Rmeileh NME. Waterpipe and cigarette tobacco smoking among Palestinian university students: a cross-sectional study. BMC Public Health 2018;18.

6 Massad SG, Shaheen M, Karam R, et al. Substance use among Palestinian youth in the West Bank, Palestine: a qualitative investigation. BMC Public Health 2016;16:800.

7 El Ansari W, Berg-Beckhoff G. Country and gender-specific achievement of healthy nutrition and physical activity guidelines: latent class analysis of 6266 university students in Egypt, Libya, and Palestine. Nutrients 2017:9:738.

8 Abukhdeir HF, Caplan LS, Reese L, et al. Factors affecting the prevalence of chronic diseases in Palestinian people: an analysis of data from the Palestinian central Bureau of statistics. East Mediterr Health J 2013;19:307-13.

9 Mensah GA, Collins PY, PYJGh C. Understanding mental health for the prevention and control of cardiovascular diseases. Glob Heart 2015;10:221.

10 SaadAdeen SAM, Battat MMK. Anxiety disorders and PTSD in Palestine: a systematic review.

11 Marie M, Hannigan B, Jones A. Mental health needs and services in the West bank, Palestine. Int J Ment Health Syst 2016;10:23.

12 Verma M, Grover S, Tripathy JP, et al. Co-Existing non-communicable diseases and mental illnesses amongst the elderly in Punjab, India. Eur Endocrinol 2019;15:106.

13 Udedi M, Pence B, Kauye F, et al. The effect of depression management on diabetes and hypertension outcomes in low- and middle-income countries: a systematic review protocol. Syst Rev 2018;7:223.

14 Allabadi $\mathrm{H}$, Alkaiyat $\mathrm{A}$, Alkhayyat $\mathrm{A}$, et al. Depression and anxiety symptoms in cardiac patients: a cross-sectional hospital-based study in a Palestinian population. BMC Public Health 2019;19:232.

15 Mittelmark MB, Sagy S, Eriksson M, et al. The Handbook of salutogenesis. Springer, 2017.

16 Simonelli F, Guerreiro AIF, Simonelli I. Six assertions about the salutogenic approach and health promotion 2010;7.

17 Posadzki P, Glass N. Self-efficacy and the sense of coherence: narrative review and a conceptual synthesis. ScientificWorldJournal 2009;9:924-33.

18 Mato M, Tsukasaki K, KJGhp T. Factors promoting sense of coherence among university students in urban areas of Japan: individual-level social capital, self-efficacy, and mental health. Glob Health Promot 2019;26:60-8.

19 Mato M, Tsukasaki K, KJHpi T. Modeling the factors associating with health-related habits among Japanese students. Health Promot Int 2019;34:300-11.

20 Kritsotakis G, Chatzi L, Vassilaki M, et al. Social capital, tolerance of diversity and adherence to Mediterranean diet: the Rhea MotherChild cohort in Crete, Greece. Public Health Nutr 2015;18:1300-7.

21 Ehsan A, Klaas HS, Bastianen A, et al. Social capital and health: a systematic review of systematic reviews. SSM Popul Health 2019;8:100425.

22 Bassett E, Moore S. Social capital and depressive symptoms: the association of psychosocial and network dimensions of social capital with depressive symptoms in Montreal, Canada. Soc Sci Med 2013;86:96-102.

23 Wilmot NA, Dauner KN. Examination of the influence of social capital on depression in fragile families. J Epidemiol Community Health 2017;71:296-302.

24 Gilbert KL, Quinn SC, Goodman RM, et al. A meta-analysis of social capital and health: a case for needed research. $J$ Health Psychol 2013;18:1385-99.

25 Hommerich C, Tiefenbach T, TJJoHS T. Analyzing the relationship between social capital and subjective well-being: the mediating role of social affiliation. J Happiness Stud 2018;19:1091-114.

26 De Silva MJ, McKenzie K, Harpham T, et al. Social capital and mental illness: a systematic review. J Epidemiol Community Health 2005;59:619-27.

27 Han K-M, Han C, Shin C, et al. Social capital, socioeconomic status, and depression in community-living elderly. J Psychiatr Res 2018;98:133-40.

28 Trap R, Rejkjær L, EHJHpi H. Empirical relations between sense of coherence and self-efficacy. National Danish Survey 2015;31:635-43.

29 Luszczynska A, Gutiérrez-Doña B, Schwarzer R. General selfefficacy in various domains of human functioning: evidence from five countries. Int J Psychol 2005;40:80-9.
30 O'Malley AJ, Christakis NA. Longitudinal analysis of large social networks: estimating the effect of health traits on changes in friendship ties. Stat Med 2011;30:950-64.

31 Christakis NA, Fowler JH. Social contagion theory: examining dynamic social networks and human behavior. Stat Med 2013;32:556-77.

32 Christakis NA, Fowler $\mathrm{JH}$. The spread of obesity in a large social network over 32 years. N Engl J Med 2007;357:370-9.

33 Fowler JH, Christakis NA. Dynamic spread of Happiness in a large social network: longitudinal analysis over 20 years in the Framingham heart study. BMJ 2008;337:a2338.

34 Rosenquist JN, Fowler JH, Christakis NA. Social network determinants of depression. Mol Psychiatry 2011;16:273-81.

35 Lobelo F, Duperly J, Frank E. Physical activity habits of doctors and medical students influence their counselling practices. $\mathrm{Br} J$ Sports Med 2009;43:89-92.

36 Frank E, Dresner Y, Shani M, et al. The association between physicians' and patients' preventive health practices. CMAJ 2013;185:649-53.

37 Houalla N, Al-Jawaldeh AE, Bagchi K. Promoting a healthy diet for the WHO eastern Mediterranean region: user-friendly guide 2012 .

38 IPAQ Research Committee. Guidelines for data processing and analysis of the International Physical Activity Questionnaire (IPAQ)short and long forms 2005.

39 Smyth CA. Evaluating sleep quality in older adults: the Pittsburgh sleep quality index can be used to detect sleep disturbances or deficits. 2008;108:42-50.

40 World Health Organization. Body mass index - BMI. Available: http:// www.euro.who.int/en/health-topics/disease-prevention/nutrition/ahealthy-lifestyle/body-mass-index-bmi

41 Topp CW, Østergaard SD, Søndergaard S, et al. The WHO-5 well-being index: a systematic review of the literature. Psychother Psychosom 2015;84:167-76.

42 Lovibond SH, Lovibond PF. Manual for the depression anxiety stress scales: psychology Foundation of Australia 1996.

43 Yari A, Nadrian $\mathrm{H}$, Rashidian $\mathrm{H}$, et al. Psychometric properties of the Persian version of social capital questionnaire in Iran. Med J Islam Repub Iran 2014;28:17.

44 Sherer M, Maddux JE, Mercandante B, et al. The self-efficacy scale: construction and validation. Psychol Rep 1982;51:663-71.

45 Holmefur M, Sundberg K, Wettergren L, et al. Measurement properties of the 13-item sense of coherence scale using Rasch analysis. Qual Life Res 2015;24:1455-63.

46 Möllerberg M-L, Årestedt K, Sandgren A, et al. Adaptation and psychometric evaluation of the short version of family sense of coherence scale in a sample of persons with cancer in the palliative stage and their family members. Pall Supp Care 2020;18:24-32.

47 Teague S, Youssef GJ, Macdonald JA, et al. Retention strategies in longitudinal cohort studies: a systematic review and meta-analysis. BMC Med Res Methodol 2018;18:1-22.

48 Spiers S, Oral E, Fontham ETH, et al. Modelling attrition and nonparticipation in a longitudinal study of prostate cancer. BMC Med Res Methodol 2018;18:1-10.

49 Eisner NL, Murray AL, Eisner M, et al. A practical guide to the analysis of non-response and attrition in longitudinal research using a real data example. Int J Behav Dev 2019:43:24-34.

50 Young AF, Powers JR, Bell SL. Attrition in longitudinal studies: who do you lose? Aust N Z J Public Health 2006;30:353-61.

51 Keyes KM, Jager J, Platt J, et al. When does attrition lead to biased estimates of alcohol consumption? bias analysis for loss to follow-up in 30 longitudinal cohorts. Int J Methods Psychiatr Res 2020;29:1-9.

52 Kristman VL, Manno M, Côté P. Methods to account for attrition in longitudinal data: do they work? A simulation study. Eur J Epidemiol 2005;20:657-62.

53 Desa U. Transforming our world: the 2030 agenda for sustainable development 2016.

54 Heller O, Somerville C, Suggs LS, et al. The process of prioritization of non-communicable diseases in the global health policy arena. Health Policy Plan 2019;34:370-83.

55 Patel V, Saxena S, Lund C, et al. The Lancet Commission on global mental health and sustainable development. The Lancet 2018;392:1553-98

56 Mosleh M, Aljeesh YI, Dalal KJBocditPhsuD-ALY P. Burden of chronic disease in the Palestinian healthcare sector using Disability-Adjusted life years (DALY), Palestine 2016;13.

57 Palestinian Central Bureau of Statistics. Population, housing and establishments census 2017; 2018.

58 Brehm BJ, Summer SS, Khoury JC, et al. Health status and lifestyle habits of US medical students: a longitudinal study. Ann Med Health Sci Res 2016;6:341-7. 
59 Mazurek Melnyk B, Slevin C, Militello L, et al. Physical health, lifestyle beliefs and behaviors, and mental health of entering graduate health professional students: evidence to support screening and early intervention. J Am Assoc Nurse Pract 2016;28:204-11.

60 Tawfik DS, Profit J, Morgenthaler TI, et al, eds. Physician burnout, well-being, and work unit safety grades in relationship to reported medical errors. Mayo Clinic Proceedings. Elsevier, 2018.

61 Hall LH, Johnson J, Heyhoe J, et al. Exploring the impact of primary care physician burnout and well-being on patient care: a focus group study. J Patient Saf 2020;16:e278-83.

62 Trockel MT, Menon NK, Rowe SG, et al. Assessment of physician sleep and wellness, burnout, and clinically significant medical errors. JAMA Netw Open 2020;3:e2028111-e.

63 Zaree TY, Nazari J, Asghary Jafarabadi M, et al. Impact of psychosocial factors on occurrence of medication errors among Tehran public hospitals nurses by evaluating the balance between effort and reward. Saf Health Work 2018;9:447-53.

64 Scheepers RA, Boerebach BCM, Arah OA, et al. A systematic review of the impact of physicians' occupational well-being on the quality of patient care. Int J Behav Med 2015;22:683-98.

65 Probst-Hensch N, Tanner M, Kessler C, et al. Prevention: a costeffective way to fight the non-communicable disease epidemic. Swiss Med Wkly 2011;141.
66 Frank E, Carrera JS, Elon L, et al. Predictors of US medical students' prevention counseling practices. Prev Med 2007;44:76-81.

67 Yu Y, Yang Y, Li Z, et al. The association between medical students' lifestyles and their attitudes towards preventive counseling in different countries. BMC Public Health 2015;15:1-8.

68 Lindstrom B. Seizing the opportunity-a salutogenic approach to public health. SciELO Public Health, 2018.

69 Bandura A. Health promotion from the perspective of social cognitive theory. Psychol Health 1998;13:623-49.

70 FAJCIR M. Collective trauma, quality of life and resilience in narratives of third generation Palestinian refugee children:1-24.

71 Collier J, Kienzler HJC health. Barriers to cardiovascular disease secondary prevention care in the West bank, Palestine-a health professional perspective 2018;12:27.

72 Carlos S, Rico-Campà A, de la Fuente-Arrillaga C, et al. Do healthy doctors deliver better messages of health promotion to their patients?: data from the sun cohort study. Eur J Public Health 2020;30:438-44.

73 Polak R, Finkelstein A, Axelrod T, et al. Medical students as health coaches: implementation of a student-initiated lifestyle medicine curriculum. Isr J Health Policy Res 2017:6:1-10. 